\title{
DIGESTÃo DE ÓLEO LUBRIFICANTE ENCAPSULADO EM FORNO DE MICROONDAS COM RADIAÇÃO FOCALIZADA POR ADIÇÃO DE AMOSTRA AO REAGENTE PRÉ-AQUECIDO
}

\author{
Flavia A. Bressani, Hamilton O. Silva e Joaquim A. Nóbrega* \\ Departamento de Química, Universidade Federal de São Carlos, CP 676, 13560-970, São Carlos - SP, Brasil \\ Letícia M. Costa \\ Departamento de Química, Universidade Federal de Minas Gerais, Belo Horizonte - MG, Brasil \\ Ana Rita A. Nogueira \\ Embrapa Pecuária Sudeste, São Carlos - SP, Brasil
}

Recebido em 2/9/05; aceito em 24/3/06; publicado na web em 25/7/06

\begin{abstract}
FOCUSED-MICROWAVE-ASSISTED DIGESTION OF ENCAPSULATED LUBRICATING OILS: GRADUAL SAMPLE ADDITION TO PRE-HEATED ACID. The applicability of the recently proposed procedure based on gradual sample addition to microwave-assisted pre-heated concentrated acid is limited by the sample viscosity. In this work, lubricating oil samples with high viscosity were encapsulated and manually added to the microwave-assisted pre-heated concentrated digestion mixture. The procedure was applied for determination of $\mathrm{Al}, \mathrm{Ca}, \mathrm{Cr}, \mathrm{Cu}, \mathrm{Fe}, \mathrm{Mg}, \mathrm{Ni}, \mathrm{P}, \mathrm{Pb}, \mathrm{Si}, \mathrm{Sn}, \mathrm{Sr}, \mathrm{V}, \mathrm{W}$, and $\mathrm{Zn}$ in lubricating oil by inductively coupled plasma optical emission spectrometry (ICP OES). Determined and certified values for $\mathrm{Ca}, \mathrm{Mg}, \mathrm{P}$, and $\mathrm{Zn}$ in lubricating oil were in agreement at a $95 \%$ confidence level.
\end{abstract}

Keywords: encapsulated lubricating oil; sample addition; focused-microwave.

\section{INTRODUÇÃO}

Procedimentos de digestão assistidos por radiação microondas têm sido implementados em laboratórios de preparo de amostras como uma alternativa rápida e segura aos procedimentos convencionais de digestão ${ }^{1}$.

A tecnologia atual pode ser dividida em sistemas com cavidade, que geralmente são operados sob altas pressão e temperatura, e em sistemas com radiação focalizada, que freqüientemente envolvem operação à pressão atmosférica. Mesmo considerando-se a disseminação desses procedimentos demonstrada em artigos de revisão ${ }^{2-4}$, novas estratégias estão sendo desenvolvidas usando fornos de microondas com radiação focalizada ${ }^{5-8}$.

Recentemente foi proposto um procedimento alternativo de digestão que se baseia na adição gradual de amostras líquidas, de baixa viscosidade, sobre o ácido aquecido por ação da radiação microondas em um forno com focalização ${ }^{9,10}$. Esse procedimento possibilita que maiores volumes de amostras sejam digeridos com menores volumes de ácidos, reduzindo a acidez final do digerido e possibilitando menor diluição para sua introdução em técnicas com nebulizadores pneumáticos. Além disso, ao programar a adição de alíquotas de amostra ao reagente concentrado e aquecido, sempre haverá um excesso de reagente disponível mantido sob alta temperatura, elevando a capacidade de formação de radicais com maior poder de oxidação da matéria orgânica. Para digestão de $2 \mathrm{~mL}$ de óleo diesel, os volumes dos ácidos utilizados foram reduzidos de 19,0 para 5,0 mL de $\mathrm{HNO}_{3}$ e de 10,0 para 1,0 $\mathrm{mL}$ de $\mathrm{H}_{2} \mathrm{SO}_{4}$ quando comparados ao procedimento convencional para digestão de óleo combustível. O tempo total do programa de aquecimento também foi reduzido em $10 \mathrm{~min}^{9}$.

Contudo, a utilização do procedimento de adição de amostras ao reagente aquecido se limita a amostras com baixa viscosidade, considerando-se o sistema de transferência de soluções do forno de

*e-mail:djan@power.ufscar.br microondas com radiação focalizada ${ }^{9}$. Para amostras com alta viscosidade, a aplicação do procedimento foi limitada pela dificuldade de aspiração e transferência para o frasco de digestão do forno de microondas quando foi utilizado dispositivo comercialmente disponível, que emprega bomba tipo seringa.

Óleos lubrificantes apresentam alta viscosidade e longas cadeias de hidrocarbonetos alifáticos e aromáticos; podem ser classificados como material de difícil digestão empregando procedimentos convencionais ou assistidos por radiação microondas. Óleos lubrificantes novos e usados são freqüentemente analisados visando a determinação de sua formulação, da concentração de metais pesados e dos metais utilizados como aditivos ${ }^{11}$. A determinação de metais em óleos naturais, óleos combustíveis e várias outras frações de petróleo é importante para uma correta avaliação do impacto ambiental das refinarias, além de proporcionar uma avaliação preventiva do desgaste de componentes mecânicos.

A ocorrência de metais em óleos naturais e lubrificantes devese principalmente a três fontes: desgaste de componentes mecânicos, contaminação e incorporação de elementos metálicos como aditivos. $\mathrm{O}$ desgaste de componentes mecânicos é produto da deterioração por fricção ou por corrosão e está diretamente relacionado à composição da maquinaria. Os contaminantes originam-se de sujeira, vazamentos, e algumas vezes, de partículas residuais de metais $^{12}$. Os aditivos são incorporados aos óleos lubrificantes para melhorar sua cor, viscosidade, ponto de fluidez, capacidade antidesgaste e propriedades anti-corrosivas e anti-oxidativas ${ }^{13}$.

Para evitar problemas associados à composição da matriz na análise de óleos e frações de petróleo quando métodos diretos de análise são empregados, procedimentos de digestão convencionais ou assistidos por radiação microondas podem ser implementados permitindo a calibração com soluções aquosas para determinação dos analitos de interesse nessas matrizes. O uso de padrões inorgânicos solúveis em água para preparo das soluções de referência para a calibração instrumental em técnicas espectrométricas, como ICP OES ou ICP-MS, apresenta vantagens, além de diminuir o custo do procedimento. 
Neste trabalho foi desenvolvido um procedimento de digestão para amostras de óleos lubrificantes, previamente encapsuladas, através da adição gradual da amostra ao reagente ácido aquecido em forno de microondas com radiação focalizada para a determinação elementar por ICP OES com visão axial.

\section{PARTE EXPERIMENTAL}

\section{Instrumentação}

Para determinação de $\mathrm{Al}, \mathrm{Ca}, \mathrm{Cr}, \mathrm{Cu}, \mathrm{Fe}, \mathrm{Mg}, \mathrm{Ni}, \mathrm{P}, \mathrm{Pb}, \mathrm{Si}, \mathrm{Sn}$, $\mathrm{Sr}$, V, W e Zn nos digeridos ácidos, foi utilizado um espectrômetro de emissão óptica com plasma acoplado indutivamente com configuração axial (Vista AX, Varian Mulgrave, Austrália). A determinação dos teores de carbono residual nos digeridos também foi efetuada por ICP OES, de acordo com o procedimento desenvolvido por Gouveia et $a l .{ }^{14}$. Um nebulizador com ranhura em V e câmara de nebulização Sturman-Masters foram empregados para introdução das amostras. A Tabela 1 apresenta as condições instrumentais utilizadas.

A digestão das amostras de óleo lubrificante foi realizada no forno de microondas com radiação focalizada com seis cavidades e operando à pressão atmosférica (STAR 6, CEM, Matthews, N.C., EUA). Frascos de digestão de quartzo foram utilizados em todos os experimentos.

\section{Reagentes, soluções e amostras}

Todas as soluções foram preparadas com reagentes de grau analítico e os volumes finais ajustados com água destilada e desionizada (Millipore, Bedford, MA, EUA). Todas as vidrarias foram previamente descontaminadas em banhos contendo $10 \% \mathrm{v} / \mathrm{v} \mathrm{HNO}_{3}$ por 24 h. Soluções de referência foram preparadas a partir de soluções estoque contendo $1000 \mathrm{mg} / \mathrm{L}$ de $\mathrm{Al}, \mathrm{Ca}, \mathrm{Cr}, \mathrm{Cu}, \mathrm{Fe}, \mathrm{Mg}, \mathrm{Ni}, \mathrm{P}, \mathrm{Pb}$, Si, Sn, Sr, V, W e Zn (Spex Sample Preparation, Metuchen, NJ, EUA), no mesmo meio dos digeridos ácidos. As soluções de refe-

Tabela 1. Parâmetros instrumentais para as medidas por ICP OES com configuração axial

\begin{tabular}{lc}
\hline Potência aplicada $(\mathrm{kW})$ & 1,3 \\
Gerador de rádio-frequiência $(\mathrm{MHz})$ & 40 \\
Vazão do gás do plasma $\left(\mathrm{L} \mathrm{min}^{-1}\right)$ & 15,0 \\
Vazão do gás auxiliar $\left(\mathrm{L} \mathrm{min}^{-1}\right)$ & 1,5 \\
Vazão do gás de nebulização $\left(\mathrm{L} \mathrm{min}^{-1}\right)$ & 0,7 \\
Padrão interno & $\mathrm{Y}$ \\
Linhas espectrais $(\mathrm{nm})$ & \\
C I & 193,025 \\
$\mathrm{Al}$ I & 396,152 \\
$\mathrm{Ca}$ II & 396,847 \\
Cr II & 205,560 \\
Cu I & 327,395 \\
Fe II & 238,204 \\
Mg I & 285,213 \\
Ni II & 221,648 \\
P I & 177,432 \\
Pb II & 220,353 \\
Si I & 250,690 \\
Sn II & 198,927 \\
Sr II & 407,771 \\
V II & 311,837 \\
W II & 207,912 \\
Zn II & 202,548 \\
Y II & 371,029 \\
\hline & \\
&
\end{tabular}

rência para calibração do ICP OES visando a determinação de carbono residual foram preparadas a partir de solução estoque 5000 $\mathrm{mg} / \mathrm{L}$ de uréia $\left(\mathrm{CH}_{4} \mathrm{~N}_{2} \mathrm{O}\right.$, Reagen, RJ, Brasil), em água desionizada. Como padrão interno, adicionou-se $1,0 \mathrm{mg} / \mathrm{L}$ de ítrio aos digeridos e às soluções de referência.

Para digestão das amostras foram utilizados $\mathrm{HNO}_{3}$ concentrado (Merck, Alemanha), $\mathrm{H}_{2} \mathrm{SO}_{4}$ concentrado (Mallinckrodt, Cidade do México, México) e $\mathrm{H}_{2} \mathrm{O}_{2} 30 \% \mathrm{~m} / \mathrm{v}$ (Merck).

Uma amostra de material de referência certificado (NIST SRM 1848 - óleo lubrificante, Gaithersburgh, MD, EUA) foi utilizada para avaliação da exatidão do procedimento proposto. Os experimentos foram conduzidos utilizando-se uma amostra de óleo lubrificante comercial novo contendo $80,9 \% \mathrm{~m} / \mathrm{m}$ de carbono, determinado por um analisador de carbono total (TOC 5000, Shimadzu, Japão)

\section{Procedimento convencional de digestão de óleo lubrificante em forno de microondas com radiação focalizada}

Uma alíquota contendo cerca de $1000 \mu \mathrm{L}$ (aproximadamente $600 \mathrm{mg}$ ) de óleo lubrificante foi transferida para o frasco reacional de quartzo do forno de microondas. Cada frasco foi colocado em uma das seis cavidades do equipamento e o programa de aquecimento descrito na Tabela 2 foi implementado. Adicionaram-se 10,0 $\mathrm{mL}$ de $\mathrm{HNO}_{3}$ e $10,0 \mathrm{~mL}$ de $\mathrm{H}_{2} \mathrm{SO}_{4}$ concentrados à amostra. Na primeira etapa de aquecimento, a temperatura foi elevada até $95{ }^{\circ} \mathrm{C}$ com posterior adição de 2,0 $\mathrm{mL}$ de $\mathrm{HNO}_{3}$, em uma única alíquota. A temperatura foi elevada a $250{ }^{\circ} \mathrm{C}$ até a etapa 4 , com adição de $\mathrm{HNO}_{3}$ nas etapas 2 e 3 . Na etapa 5 , ocorreu resfriamento até $200{ }^{\circ} \mathrm{C}$ para posterior introdução gradual de $20,0 \mathrm{~mL} \mathrm{de} \mathrm{H}_{2} \mathrm{O}_{2}$. As replicatas foram realizadas na mesma cavidade ou em cavidades vizinhas, conforme discutido por Costa et al. ${ }^{15}$.

Após execução dos programas de aquecimento, os digeridos foram diluídos para um volume final de $50,0 \mathrm{~mL}$ com água destilada-desionizada e armazenados em frascos de polipropileno.

Tabela 2. Programa de aquecimento para digestão de óleo lubrificante em forno de microondas com radiação focalizada pelo procedimento convencional

Adição inicial: 10,0 mL $\mathrm{HNO}_{3}+10,0 \mathrm{~mL} \mathrm{H}_{2} \mathrm{SO}_{4}$ concentrados

\begin{tabular}{lccccc}
\hline Etapa & $\begin{array}{c}\mathrm{t}_{\text {rampa }} \\
(\mathrm{min})\end{array}$ & $\begin{array}{c}\mathrm{T} \\
\left({ }^{\circ} \mathrm{C}\right)\end{array}$ & $\mathrm{t}_{\text {patamar }}$ & $\begin{array}{c}\text { Reagente } \\
(\mathrm{mL})\end{array}$ & $\begin{array}{c}\text { Alíquota } \\
(\mathrm{mL})\end{array}$ \\
\hline 1 & 1 & 95 & $1 \mathrm{~s}$ & $2 \mathrm{HNO}_{3}$ & 2 \\
2 & 3 & 150 & $1 \mathrm{~s}$ & $2 \mathrm{HNO}_{3}$ & 2 \\
3 & 3 & 220 & $5 \mathrm{~min}$ & $5 \mathrm{HNO}_{3}$ & 1 \\
4 & 2 & 250 & 0 & - & - \\
5 & 0 & 200 & $10 \mathrm{~min}$ & $20 \mathrm{H}_{2} \mathrm{O}_{2}$ & 1 \\
\hline
\end{tabular}

Encapsulamento e procedimento alternativo de digestão de óleo lubrificante em forno de microondas com radiação focalizada

Inicialmente duas cápsulas transparentes gelatinosas disponíveis em farmácias homeopáticas foram preenchidas com cerca de $300 \mathrm{mg}$ de óleo lubrificante.

No frasco reacional de vidro borossilicato do forno de microondas foi adicionada uma mistura ácida contendo $4 \mathrm{~mL}$ de $\mathrm{HNO}_{3} \mathrm{e}$ $3 \mathrm{~mL}$ de $\mathrm{H}_{2} \mathrm{SO}_{4}$ concentrados. Usando a bomba tipo seringa do equipamento, 2 alíquotas de $1,5 \mathrm{~mL}$ de $\mathrm{HNO}_{3}$ foram adicionadas ao longo do programa de aquecimento. Na última etapa programou-se a adição de alíquotas de $1 \mathrm{~mL} \mathrm{H}_{2} \mathrm{O}_{2} /$ min totalizando um volume de $10 \mathrm{~mL}$ de $\mathrm{H}_{2} \mathrm{O}_{2}$. As cápsulas contendo o óleo lubrificante foram 
manualmente adicionadas sobre a mistura ácida aquecida, conforme o programa apresentado na Tabela 3 .

ATENÇÃO: Não se recomenda o emprego desse procedimento em frascos abertos aquecidos em chapas elétricas ou banhos de areia. A adição da amostra à mistura digestora contendo ácidos concentrados aquecidos implica em uma reação abrupta e intensa que pode oferecer riscos ao analista. O forno de microondas com radiação focalizada tem dispositivos de segurança que possibilitam menores riscos ao analista. Alternativamente, poder-se-ia construir uma haste de vidro associada a um motor de passo que possibilitasse a transferência mecânica da cápsula contendo a amostra para a mistura digestora.

Tabela 3. Programa de aquecimento para a digestão das amostras de óleo lubrificante em forno de microondas com radiação focalizada pelo procedimento alternativo de adição de amostras encapsuladas sobre a mistura ácida aquecida

Adição inicial: 4,0 mL $\mathrm{HNO}_{3}+3,0 \mathrm{~mL} \mathrm{H}_{2} \mathrm{SO}_{4}$ concentrados

\begin{tabular}{lccccc}
\hline Etapa & $\begin{array}{c}\mathrm{t}_{\text {rampa }} \\
(\mathrm{min})\end{array}$ & $\begin{array}{c}\mathrm{T} \\
\left({ }^{\circ} \mathrm{C}\right)\end{array}$ & $\begin{array}{c}\mathrm{t}_{\text {patamar }} \\
(\mathrm{min})\end{array}$ & Adição & Alíquota \\
\hline 1 & 1 & 130 & 2 & 2 cápsulas & 1 cápsula $/ \mathrm{min}$ \\
2 & 3 & 150 & 6 & $3,0 \mathrm{~mL} \mathrm{HNO} \mathrm{HNO}_{3}$ & $1,5 \mathrm{~mL} / 2 \mathrm{~min}$ \\
3 & 3 & 180 & 10 & $10,0 \mathrm{~mL} \mathrm{H} \mathrm{O}_{2}$ & $1,0 \mathrm{~mL} / \mathrm{min}$ \\
\hline
\end{tabular}

O digerido final foi diluído para $15 \mathrm{~mL}$ com água desionizada. Posteriormente, esse digerido foi diluído $1: 5 \mathrm{v} \mathrm{v}^{-1}$ para determinação do $\mathrm{C}$ residual e de elementos presentes em baixas concentrações. Uma diluição $1: 10 \mathrm{v} \mathrm{v}^{-1}$ foi efetuada para determinação de elementos presentes em maiores concentrações, tais como $\mathrm{Mg}$ e $\mathrm{P}$.

O branco analítico foi realizado pela digestão de 2 cápsulas de gelatina para avaliar eventual contaminação causada por elementos presentes neste material. Esses digeridos também foram determinados por ICP OES.

A acidez final de todos os digeridos foi determinada por titulação ácido-base utilizando solução padronizada de $\mathrm{NaOH}$.

Visando-se ter uma idéia preliminar dos possíveis efeitos de fatores experimentais facilmente controláveis no procedimento, tais como massa de amostra, número de alíquotas e temperatura de adição, sobre a eficiência da digestão foi realizado um planejamento fatorial $2^{3}$. Buscou-se verificar qual a influência das variáveis selecionadas sobre a acidez dos digeridos e os teores de carbono residual. Os níveis selecionados estão descritos na Tabela 4.

Tabela 4. Níveis dos fatores estabelecidos para o planejamento experimental considerando a digestão em forno de microondas com radiação focalizada

\begin{tabular}{lccc}
\hline Variáveis & $\begin{array}{c}\text { Inferior } \\
(-)\end{array}$ & $\begin{array}{c}\text { Superior } \\
(+)\end{array}$ & $\begin{array}{c}\text { Ponto médio } \\
(0)\end{array}$ \\
\hline Massa da amostra (mg) & 200 & 600 & 400 \\
Número de alíquotas & 1 & 3 & 2 \\
Temperatura da adição $\left({ }^{\circ} \mathrm{C}\right)$ & 130 & 150 & 140 \\
\hline
\end{tabular}

\section{RESULTADOS E DISCUSSÃO}

Procedimentos convencionais de digestão de amostras com alto teor de compostos orgânicos, tais como os óleos lubrificantes, geralmente utilizam grandes volumes de reagentes ácidos para uma destruição da matéria orgânica mais efetiva, garantindo assim baixos teores de carbono residual.
Comparando-se o procedimento convencional (Tabela 2) e o procedimento desenvolvido (Tabela 3 ) para a digestão de óleo, observase uma redução de 19,0 para 7,0 $\mathrm{mL}$ de $\mathrm{HNO}_{3}$, de 10,0 para $3,0 \mathrm{~mL}$ de $\mathrm{H}_{2} \mathrm{SO}_{4}$ e de 20,0 para $10,0 \mathrm{~mL}$ de $\mathrm{H}_{2} \mathrm{O}_{2}$ para a digestão de aproximadamente $600 \mathrm{mg}$ de óleo lubrificante. $\mathrm{O}$ encapsulamento de alíquotas de óleo lubrificante possibilitou a fácil introdução sucessiva de alíquotas das amostras aos ácidos nítrico e sulfúrico concentrados e aquecidos por ação da radiação microondas. $\mathrm{O}$ procedimento foi facilmente implementado no forno de microondas com radiação focalizada, sem a necessidade de modificações no equipamento. As duas alíquotas das amostras encapsuladas foram introduzidas na mistura ácida aquecida em intervalos de 1 min (Tabela 3 - Etapa 1). Esse intervalo de tempo possibilitou uma digestão prévia parcial da primeira alíquota anteriormente à adição da segunda alíquota aumentando assim, a eficiência do processo de digestão.

Os teores de carbono residual para os digeridos produzidos após a adição gradual das amostras encapsuladas variaram de 0,68 a $3,07 \%$, enquanto que para os digeridos obtidos após aplicação do procedimento convencional de digestão os teores de carbono residual determinados foram superiores a $2,89 \%$.

A eficiência de digestão obtida utilizando-se o procedimento de digestão através da adição gradual de amostra encapsulada ao reagente aquecido por radiação microondas demonstra a aplicabilidade do procedimento alternativo para amostras com altos teores de matéria orgânica e alta viscosidade. Outro ponto relevante é a baixa acidez residual dos digeridos, já que um menor volume de ácidos concentrados foi utilizado, possibilitando a menor diluição previamente à introdução dessas soluções em equipamentos com nebulização pneumática. Comparando-se as concentrações ácidas dos digeridos para ambos os procedimentos, observou-se que os digeridos obtidos pelo procedimento alternativo tiveram acidez residual inferior a 2,0 mol $\mathrm{L}^{-1}$ e os digeridos obtidos pelo procedimento convencional apresentaram acidez superior a $4,0 \mathrm{~mol} \mathrm{~L}^{-1}$.

A matriz de dados resultante do planejamento fatorial $2^{3}$ está apresentada na Tabela 5. Segundo os gráficos de Pareto obtidos a partir do planejamento fatorial não houve influência significativa dos fatores estudados sobre o teor de carbono residual (Figura 1). Observa-se que a temperatura e a massa da amostra influenciaram significativamente o processo quando a acidez do digerido foi avaliada (Figura 2). Menores valores de acidez foram obtidos quando a massa de $600 \mathrm{mg}$ de amostra foi adicionada sobre a mistura ácida aquecida a $150{ }^{\circ} \mathrm{C}$. Entretanto, não houve diferenças apreciáveis nos teores de carbono residual quando a massa da amostra foi variada de 200 a $600 \mathrm{mg}$. Pode-se supor que as condições de digestão, i.e., temperatura, tempo e concentração dos reagentes, são suficientes para proporcionar uma oxidação eficiente

Tabela 5. Matriz de dados do planejamento fatorial $2^{3}$ : acidez do digerido e teor de carbono residual (RCC)

\begin{tabular}{lccccc}
\hline Experimento Massa & Alíquotas & Temperatura & $\begin{array}{c}\text { Acidez } \\
\left(\mathrm{mol} \mathrm{L}^{-1}\right)\end{array}$ & $\begin{array}{c}\mathrm{RCC} \\
\left(\% \mathrm{~m} \mathrm{v}^{-1}\right)\end{array}$ \\
\hline 1 & + & + & + & 0,720 & 2,62 \\
2 & + & + & - & 1,09 & 2,26 \\
3 & + & - & + & 0,550 & 2,49 \\
4 & + & - & - & 1,07 & 2,45 \\
5 & - & + & + & 0,970 & 0,68 \\
6 & - & + & - & 1,22 & 2,37 \\
7 & - & - & + & 1,16 & 2,77 \\
8 & - & - & - & 1,24 & 2,69 \\
9 & 0 & 0 & 0 & 1,13 & 2,14 \\
10 & 0 & 0 & 0 & 1,17 & 2,32 \\
11 & 0 & 0 & 0 & 1,16 & 3,07 \\
\hline
\end{tabular}




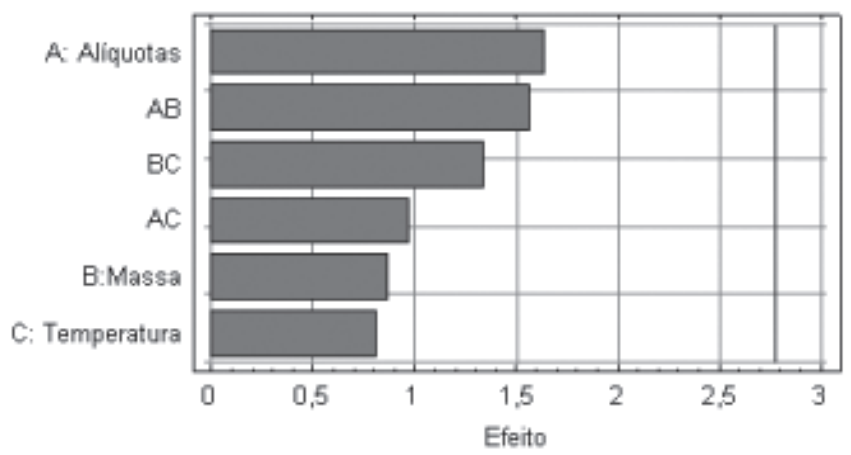

Figura 1. Gráfico de Pareto - Influência dos fatores selecionados sobre o teor de carbono residual (RCC) dos digeridos obtidos com as alíquotas de óleo encapsuladas adicionadas sobre o ácido aquecido com o forno de microondas com radiação focalizada

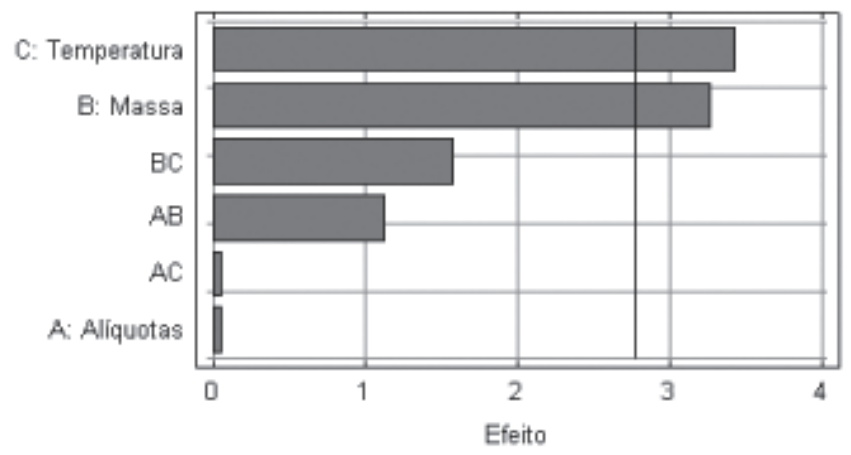

Figura 2. Gráfico de Pareto - Influência dos fatores selecionados sobre a acidez dos digeridos obtidos com as alíquotas de óleo encapsuladas adicionadas sobre o ácido aquecido com o forno de microondas com radiação focalizada

mesmo quando se utilizou maior massa de amostra e que ainda seria possível digerir massas superiores de óleo lubrificante nas condições propostas. Contudo, a investigação desse aspecto não foi um objetivo do experimento fatorial. Provavelmente as condições drásticas de operação tenham eliminado o possível efeito do número de alíquotas da amostra gradualmente adicionadas sobre o processo de digestão. Além disso, pode-se supor que a variação de temperatura na faixa de 130 a $150{ }^{\circ} \mathrm{C}$ não possibilitou discriminar o efeito dessa variável. $\mathrm{O}$ uso de menor temperatura de adição das amostras demonstraria mais claramente o efeito dessa variável, porém experimentos sob temperatura de adição de $100{ }^{\circ} \mathrm{C}$ não foram bem sucedidos pois foram observados resíduos sólidos após o final da digestão.

Com relação ao branco analítico obtido digerindo-se apenas as cápsulas gelatinosas, apenas $\mathrm{Ca}$ e $\mathrm{Mg}$ foram determinados em concentrações relativamente baixas, i.e. aproximadamente $1,07 \mu \mathrm{g} \mathrm{g}^{-1}$ de $\mathrm{Ca}$ e $0,177 \mu \mathrm{g} \mathrm{g}^{-1}$ de $\mathrm{Mg}$.

A determinação dos elementos nos digeridos das amostras de óleo lubrificante de mesma marca quando novo (A) e quando usado no mesmo carro que percorreu $800 \mathrm{~km}$ (A1) e $2875 \mathrm{~km}$ (A2), nas amostras de óleos usados de diferentes postos de gasolina da região (B, C e D) e no material de referência certificado foi efetuada por ICP OES com configuração axial e os resultados estão apresentados nas Tabelas 6, 7 e 8, respectivamente. Observa-se um aumento das concentrações de $\mathrm{Al}, \mathrm{Cu}$ e Fe com o aumento da distância percorrida pelo automóvel. Sabe-se que as concentrações desses elementos podem variar com a distância percorrida, com o uso do filtro de óleo do carro e com o desgaste das partes metálicas do automóvel. O percurso máximo avaliado, $2875 \mathrm{~km}$, não foi sufici- ente para alterar a concentração da maioria dos elementos que são adicionados ao óleo novo, provavelmente na forma de aditivos, no processo de fabricação. Não há diferença significativa nos teores de $\mathrm{Ca}, \mathrm{P}$ e Zn, sendo que apenas se pôde observar uma diminuição no teor de $\mathrm{Mg}$, provavelmente devido ao processo de lubrificação. Para diferentes tipos de óleos usados (Tabela 7) observaram-se maiores quantidades de elementos provenientes de aditivos, como $\mathrm{Ca}, \mathrm{P}$ e Zn. Magnésio também foi encontrado em menores concentrações quando comparado ao óleo lubrificante novo. Os teores de $\mathrm{Cr}, \mathrm{Ni}$,

Tabela 6. Elementos determinados nos digeridos de óleo lubrificante quando novo (A) e quando usado em carro à gasolina que percorreu $800 \mathrm{~km}$ (A1) e $2875 \mathrm{~km}$ (A2), (média \pm desvio padrão, $\mathrm{n}=3$ )

\begin{tabular}{lccc}
\hline Elementos & $\mathrm{A}\left(\mu \mathrm{g} \mathrm{g}^{-1}\right)$ & $\mathrm{A} 1\left(\mu \mathrm{g} \mathrm{g}^{-1}\right)$ & $\mathrm{A} 2\left(\mu \mathrm{g} \mathrm{g}^{-1}\right)$ \\
\hline $\mathrm{Al}$ & $4,7 \pm 0,4$ & $7,7 \pm 0,3$ & $20,0 \pm 2,9$ \\
$\mathrm{Ca}$ & $140,8 \pm 16,0$ & $118,1 \pm 8,2$ & $117,8 \pm 19,3$ \\
$\mathrm{Cr}$ & $<0,258$ & $<0,258$ & $<0,258$ \\
$\mathrm{Cu}$ & $<0,003$ & $1,6 \pm 0,1$ & $7,2 \pm 0,4$ \\
$\mathrm{Fe}$ & $4,7 \pm 0,5$ & $37,5 \pm 8,8$ & $88,9 \pm 3,0$ \\
$\mathrm{Mg}$ & $1026,3 \pm 28,4$ & $767,1 \pm 18,0$ & $842,9 \pm 11,3$ \\
$\mathrm{Ni}$ & $<0,004$ & $<0,004$ & $<0,004$ \\
$\mathrm{P}$ & $916,2 \pm 24,4$ & $981,4 \pm 24,1$ & $922,7 \pm 9,9$ \\
$\mathrm{~Pb}$ & $<0,009$ & $<0,009$ & $<0,009$ \\
$\mathrm{Si}$ & $<0,001$ & $<0,001$ & $<0,001$ \\
$\mathrm{Sn}$ & $<0,321$ & $<0,321$ & $<0,321$ \\
$\mathrm{Sr}$ & $<0,031$ & $<0,031$ & $<0,031$ \\
$\mathrm{~V}$ & $<0,001$ & $<0,001$ & $<0,001$ \\
$\mathrm{~W}$ & $<0,015$ & $<0,015$ & $<0,015$ \\
$\mathrm{Zn}$ & $869,9 \pm 29,9$ & $606,6 \pm 7,3$ & $740,8 \pm 3,7$ \\
\hline
\end{tabular}

Tabela 7. Elementos determinados nos digeridos de óleos lubrificantes usados de diferentes postos de gasolina (média \pm desvio padrão, $\mathrm{n}=3$ )

\begin{tabular}{lccc}
\hline Elementos & $\mathrm{B}\left(\mu \mathrm{g} \mathrm{g}^{-1}\right)$ & $\mathrm{C}\left(\mu \mathrm{g} \mathrm{g}^{-1}\right)$ & $\mathrm{D}\left(\mu \mathrm{g} \mathrm{g}^{-1}\right)$ \\
\hline $\mathrm{Al}$ & $14,2 \pm 2,8$ & $10,1 \pm 1,0$ & $25,9 \pm 1,4$ \\
$\mathrm{Ca}$ & $3687,9 \pm 98,9$ & $938,8 \pm 11,4$ & $499,8 \pm 24,2$ \\
$\mathrm{Cr}$ & $<0,258$ & $<0,258$ & $<0,258$ \\
$\mathrm{Cu}$ & $95,1 \pm 1,8$ & $8,7 \pm 1,0$ & $26,3 \pm 0,7$ \\
$\mathrm{Fe}$ & $99,0 \pm 3,8$ & $110,2 \pm 16,4$ & $142,8 \pm 9,2$ \\
$\mathrm{Mg}$ & $42,8 \pm 3,7$ & $899,5 \pm 4,6$ & $845,9 \pm 8,6$ \\
$\mathrm{Ni}$ & $<0,004$ & $<0,004$ & $<0,004$ \\
$\mathrm{P}$ & $1341,7 \pm 45,8$ & $1021,7 \pm 27,6$ & $1043,0 \pm 14,7$ \\
$\mathrm{~Pb}$ & $<0,009$ & $<0,009$ & $<0,009$ \\
$\mathrm{Si}$ & $<0,001$ & $<0,001$ & $<0,001$ \\
$\mathrm{Sn}$ & $<0,321$ & $<0,321$ & $<0,321$ \\
$\mathrm{Sr}$ & $<0,031$ & $<0,031$ & $<0,031$ \\
$\mathrm{~V}$ & $<0,001$ & $<0,001$ & $<0,001$ \\
$\mathrm{~W}$ & $<0,015$ & $<0,015$ & $<0,015$ \\
$\mathrm{Zn}$ & $1199,8 \pm 27,6$ & $874,8 \pm 23,1$ & $1013,8 \pm 9,1$ \\
\hline
\end{tabular}

Tabela 8. Teores de Ca, Mg, P e Zn (média \pm desvio padrão, $\mathrm{n}=3$ ) no material de referência certificado (NIST SRM 1848) empregandose o procedimento de adição gradual de amostra encapsulada ao reagente aquecido

\begin{tabular}{lcc}
\hline Elemento & Certificado $\left(\% \mathrm{~m} \mathrm{~m}^{-1}\right)$ & Determinado $\left(\% \mathrm{~m} \mathrm{~m}^{-1}\right)$ \\
\hline $\mathrm{Ca}$ & $0,359 \pm 0,011$ & $0,327 \pm 0,010$ \\
$\mathrm{Mg}$ & $0,821 \pm 0,038$ & $0,844 \pm 0,038$ \\
$\mathrm{P}$ & $0,788 \pm 0,028$ & $0,781 \pm 0,026$ \\
$\mathrm{Zn}$ & $0,873 \pm 0,022$ & $0,715 \pm 0,084$ \\
\hline
\end{tabular}


$\mathrm{Pb}, \mathrm{Si}, \mathrm{Sn}, \mathrm{Sr}, \mathrm{V}$ e W foram abaixo dos limites de detecção do procedimento para todas as amostras de óleo analisadas.

Para a amostra do material de referência, os resultados obtidos foram concordantes em um nível de confiança de 95\%, aplicandose o teste- $t$ de Student.

\section{CONCLUSÃO}

O encapsulamento das amostras foi uma estratégia simples que viabilizou o procedimento de adição de amostra viscosa ao reagente aquecido no forno de microondas com radiação focalizada. O procedimento foi aplicado com eficiência para amostras de óleos lubrificantes. Os digeridos obtidos caracterizaram-se por baixos teores de carbono e acidez residuais, possibilitando a introdução em equipamentos com nebulizadores pneumáticos empregando-se menores fatores de diluição. Além disso, foram necessários menores volumes de reagentes concentrados e temperatura de digestão.

\section{AGRADECIMENTOS}

À Fundação de Amparo à Pesquisa do Estado de São Paulo (FAPESP) e ao Conselho Nacional de Desenvolvimento Científico e Tecnológico $(\mathrm{CNPq})$ pelo apoio financeiro e bolsas concedidas. $\mathrm{O}$ apoio de infra-estrutura laboratorial proporcionado pelo Centro de Caracterização e Desenvolvimento de Materiais (CCDM, UFSCar-
UNESP) também foi essencial para o desenvolvimento do trabalho e os autores também expressam seus agradecimentos.

\section{REFERÊNCIAS}

1. Kingston, H. M.; Haswell, S. J., eds.; Microwave-Enhanced Chemistry. Fundamentals, Sample Preparation and Applications, American Chemical Society: Washington, 1997.

2. Nóbrega, J. A.; Trevizan, L. C.; Araújo, G. C. L.; Nogueira, A. R. A.; Spectrochim. Acta 2002, 57B, 1855.

3. Smith, F. E.; Arsenault, E. A.; Talanta 1996, 43, 1207.

4. Oliveira, E.; J. Braz. Chem. Soc. 2003, 14, 174.

5. Brancalion, M. L.; Arruda, M. A. Z.; Microchim. Acta 2005, 150, 283.

6. Araújo, G. C. L.; Nogueira, A. R. A.; Nóbrega, J. A.; The Analyst 2000, $125,1861$.

7. Trevizan, L. C.; Nogueira, A. R. A.; Nóbrega, J. A.; Talanta 2003, 61, 81.

8. Araújo, G. C. L.; Nogueira, A. R. A.; Nóbrega, J. A.; Microchim. Acta 2004, 144,81 .

9. Nóbrega, J. A.; Costa, L. M.; Santos, D. M.; Analytica 2002, 1, 32.

10. Santos, D. M.; Pedroso, M. M.; Costa, L. M.; Nogueira, A. R. A.; Nóbrega, J. A.; Talanta 2005, 65, 505.

11. Hiligoss, D.; O'Lear, D.; At. Spectrosc. 2001, 22, 276.

12. Brown, R. J.; Spectrochim. Acta 1983, 38B, 283.

13. Ekanem, E. J.; Lori, J. A.; Thomas, S. A.; Talanta 1997, 44, 2103.

14. Gouveia, S. T.; Silva, F. V.; Costa, L. M.; Nogueira, A. R. A.; Nóbrega, J. A.; Anal. Chim. Acta 2001, 445, 269.

15. Costa, L. M.; Silva, F. V.; Gouveia, S. T.; Nogueira, A. R. A.; Nóbrega, J. A.; Spectrochim. Acta 2001, 56B, 1981. 